\title{
Magnetoplasmon spectrum of a weakly modulated two-dimensional electron gas system
}

\author{
M. Tahir ${ }^{1, *}$, K. Sabeeh ${ }^{2, \dagger}$ and A. MacKinnon ${ }^{1}$ \\ ${ }^{1}$ Department of Physics, Blackett Laboratory, \\ Imperial College London, South Kensington Campus, \\ London SW7 2AZ, United Kingdom. and \\ ${ }^{2}$ Department of Physics, Quaid-i-Azam University Islamabad 45320, Pakistan.
}

(Date textdate; Received textdate; Revised textdate; Accepted textdate; Published textdate)

\begin{abstract}
The magnetoplasmon spectrum of a magnetically modulated two-dimensional electron gas (MM2DEG) is investigated. We derive the inter and intra Landau band magnetoplasmon spectrum within the self consistent field approach. The derivation is performed at zero temperature as well as at finite temperature. Results are presented for the inter and intra Landau band magnetoplasmon spectrum as a function of the inverse magnetic field. As a result of magnetic modulation, magnetic Weiss oscillations are found to occur in the magnetoplasmon spectrum. Furthermore, our finite temperature theory facilitates analysis of effects of temperature on the magnetoplasmon spectrum. The results are compared with those obtained for an electrically modulated 2DEG system. In addition, we derive and discuss the effects of simultaneous electric and magnetic modulations on the magnetoplasmon spectrum of 2DEG when the modulations are in phase as well as when they are out of phase. Magnetic oscillations are affected by the relative phase of the two modulations and position of the oscillations depends on the relative strength of the two modulations in the former case while we find complete suppression of Weiss oscillations for particular relative strength of the modulations in the latter case.
\end{abstract}




\section{MAGNETOPLASMON SPECTRUM OF MAGNETICALLY MODULATED TWO-DIMENSIONAL ELECTRON GAS (MM2DEG)}

In the past two decades, remarkable progress has been made in epitaxial crystal growth techniques which have made possible the fabrication of novel semiconductor heterostructures. These modern microstructuring techniques can be used to laterally confine quasi-twodimensional electron gas (2DEG) in e.g.,GaAs/AlGaAs heterostructure on a submicrometer scale. Furthermore, magnetic modulation of these systems can be realized by depositing an array of ferromagnetic strips on top of the heterostructure or by superconducting layers beneath the substrate. These magnetically modulated 2DEG systems realized in semiconductor heterostructures have attracted a lot of attention in the past and continue to do so


18, 19, 20, 21, 22, 23, 24, 25, 26, 27, 28]. Primarily, it is due to the introduction of another length scale in the system, the period of modulation, as a result new phenomena arise due to commensurability of the period of modulation and the other characteristic length scale of the system, cyclotron diameter at the Fermi level. Resistivity measurements on these systems are found to exhibit commensurability oscillations (magnetic Weiss oscillations) since the magnetic modulation broadens the Landau levels into minibands whose width oscillates as a function of the magnetic field. These oscillations are periodic as a function of the inverse magnetic field with a larger period than that of the Shubnikov-de Hass ( $\mathrm{SdH}$ ) oscillations. The period of Weiss oscillations depends on both the modulation period and the square root of the number density of the MM2DEG, in contrast to the linear dependence on the number density of the $\mathrm{SdH}$ oscillations. Moreover, the amplitude of Weiss oscillations is weakly affected by temperature as compared to $\mathrm{SdH}$ oscillations.

We investigate the effects of magnetic modulation on the collective excitations (magnetoplasmons) of a 2DEG. Plasmons are a very general phenomenon and have been studied extensively in a wide variety of systems including ionized gases, simple metals and semiconductor 2DEG systems. In a 2DEG, these collective excitations are induced by the electronelectron interactions. These collective excitations, plasmons, are among the most important electronic properties of a system. In the presence of an external magnetic field, these collective excitations are known as magnetoplasmons. Magnetic oscillations of the plasmon frequency occur in a magnetic field. Single particle magneto-oscillatory phenomena such 
as the Shubnikov-de Haas and de Haas-van Alphen effects have provided very important probes of the electronic structure of solids. Their collective analog yields important insights into collective phenomena. For this reason we study the effects of magnetic modulation on the collective excitations (magnetoplasmons) in a 2DEG.

We present the magnetoplasmon spectrum of a MM2DEG in the presence of a perpendicular magnetic field using the self consistent field approach. Both the inter and intra Landau band magnetoplasmons are determined. Inter Landau band magnetoplasmons arise due to electronic transitions between different Landau bands whereas intra Landau band magnetoplasmons are a result of electronic transitions in a single Landau band. We evaluate the dynamic, nonlocal density-density response function to obtain these results. The collective excitations in a MM2DEG system have been investigated in the past [12, 16, 20, 21, 24]. All of these studies primarily investigate the inter Landau band magnetoplasmon mode. Reference [16] is an experimental study where the inter Landau band magnetoplasmons are investigated by infrared optical measurements whereas [12, 20, 21, 24] are theoretical studies. In [12] only the primary inter Landau band magnetoplasmon was considered. Far-infrared absorption of MM2DEG was theoretically investigated in [20] to explore plasma oscillations in the system. Similarly plasma oscillations were also theoretically investigated in [21, 24] taking into account primarily the inter Landau band magnetoplasmons. Since the existence of an intra-Landau band magnetoplasmon is a result of the finite Landau bandwidth caused by modulation, the study of collective excitations and in particular modulation induced effects in this system requires taking into account the intra Landau band magnetoplasmons. To overcome this shortcoming in previous work mentioned above, we determine both the intra and inter-Landau band magnetoplasmon spectrum in this work. In addition, our finite temperature theory facilitates the analysis of effects of temperature on the Weiss \& SdH oscillations in the magnetoplasmon spectrum of MM2DEG. Furthermore, we carry out a detailed comparison of phase and amplitude of magnetic Weiss oscillations and the electric Weiss oscillations in a 2DEG. We also present the effects of simultaneous electric and magnetic modulations on the magnetoplasmon spectrum of a 2DEG. To the best of our knowledge, the complete study of both the inter and intra Landau band magnetoplasmon spectrum in this system and comparison of these with the results for the electrically modulated two-dimensional electron gas system (EM2DEG) [29, 30, 31, 32] has not been carried out so far. To this end, we have undertaken the present study. 
In section II, we present the formulation of the problem. Section III contains the magnetoplasmon spectrum of a MM2DEG and comparison with an EM2DEG at zero temperature $(T=0)$ whereas in section IV we discuss the temperature dependence of magnetoplasmon spectrum of a MM2DEG and its comparison with an EM2DEG including the asymptotic description. In the following two sections, magnetoplasmon spectrum in the presence of simultaneous electric and magnetic modulations is presented. We discuss the effects of inphase modulations (electric and magnetic) in section $\mathrm{V}$ while out of phase is discussed in section VI. Concluding remarks are made in section VII.

\section{FORMULATION}

The system that we are considering is a $2 \mathrm{DEG}$ in the presence of a perpendicular magnetic field that is modulated weakly and periodically along one direction. We take the magnetic field $B$ to be perpendicular to the $x-y$ plane in which electrons with unmodulated areal density $n_{D}$, effective mass $m^{*}$ and charge $-e$ are confined. We employ the Landau gauge and write the vector potential as $A=\left(0, B x+\left(B_{0} / K\right) \sin K x, 0\right)$, where $K$ is $2 \pi / a, a$ is the period of modulation and $B_{0}$ is the magnetic modulation strength such that $B_{0} \ll B$. The Hamiltonian in the Landau gauge is [5, 8, 26]

$$
H_{0}=\frac{1}{2 m^{*}}\left[-\hbar^{2} \frac{\partial^{2}}{\partial x^{2}}+\left(-i \hbar \frac{\partial}{\partial y}+e B x+\left(e B_{0} / K\right) \sin K x\right)^{2}\right] .
$$

Since the Hamiltonian does not depend on the $y$ coordinate, the unperturbed wavefunctions are plane waves in the the $y$-direction. This allows us to write for the wavefunctions,

$$
\phi_{n k_{y}}(\bar{x})=\frac{1}{\sqrt{L_{y}}} e^{i k_{y} y} u_{n}(x),
$$

with $L_{y}$ being a normalization length in $y$-direction and $\bar{x}$ a $2 \mathrm{D}$ position vector in the $x-y$ plane. The hamiltonian in equation (1) can be expressed as

$$
\begin{aligned}
H_{0} & =-\frac{\hbar^{2}}{2 m^{*}} \frac{\partial^{2}}{\partial x^{2}}+\frac{1}{2} m^{*} \omega_{c}^{2}\left(x-x_{0}\right)^{2} \\
& +\left(\omega_{0} / K\right)\left(p_{y}+e B x\right) \sin K x+\left(m^{*} \omega_{0}^{2} / 4 K^{2}\right)[1-\cos 2 K x],
\end{aligned}
$$

where $\omega_{c}=\frac{e B}{m^{*}}$ is the cyclotron frequency, $\omega_{0}=e B_{0} / m^{*}$ is the modulation frequency,

$x_{0}=-l^{2} k_{y}=-\frac{\hbar k_{y}}{m^{*} \omega_{c}}$ is the coordinate of cyclotron orbit center, $l=\sqrt{\frac{\hbar}{m^{*} \omega_{c}}}$ is the magnetic length, and $m^{*}$ is the effective mass. We can write the unmodulated eigenstates in the form 
$\phi_{n k_{y}}(\bar{x})=\frac{1}{\sqrt{L_{y}}} e^{i k_{y} y} u_{n}\left(x ; x_{0}\right)$, with $u_{n}\left(x ; x_{0}\right)=\left(\sqrt{\pi} 2^{n} n ! l\right)^{\frac{-1}{2}} \exp \left(-\frac{1}{2 l^{2}}\left(x-x_{0}\right)^{2}\right) H_{n}\left(\frac{x-x_{0}}{l}\right)$, where $u_{n}\left(x ; x_{0}\right)$ is a normalized harmonic oscillator wavefunction centered at $x_{0}$ and $H_{n}(x)$ are Hermite polynomials with $n$ the Landau level quantum number[33]. Since we are considering weak modulation $B_{0}<<B$, we can apply standard perturbation theory to determine the first order corrections to the unmodulated energy eigenvalues in the presence of modulation

$$
\varepsilon\left(n, x_{0}\right)=(n+1 / 2) \hbar \omega_{c}+G_{n} \cos \left(\frac{2 \pi}{a} x_{0}\right)
$$

where $G_{n}=\hbar \omega_{0} \exp (-u / 2)\left[L_{n}(u) / 2+L_{n-1}^{1}(u)\right], u=\frac{K^{2} l^{2}}{2}=\left(\frac{2 \pi}{a}\right)^{2} \frac{\hbar}{2 m^{*} \omega_{c}}$ and $L_{n}(u)$, $L_{n-1}^{l}(u)$ are Laguerre and associated Laguerre polynomials. This result has been obtained previously [5, 8]. The above equation shows that the formerly sharp Landau levels are now broadened into minibands by the modulation potential. Furthermore, the Landau bandwidth $\left(\sim\left|G_{n}\right|\right)$ oscillates as a function of $n$, since $L_{n}(u)$ is an oscillatory function of its index [33].

Hereafter, we employ the Ehrenreich-Cohen Self-Consistent Field (SCF) approach to determine the density-density response function[34]. Following the $\mathrm{SCF}$ approach, $\Pi_{0}(\bar{q}, \omega)$ is the density-density response function of the non-interacting electron system, given by

$$
\Pi_{0}(\bar{q}, \omega)=\frac{1}{A} \sum_{n, n^{\prime}} \sum_{k_{y}} C_{n n^{\prime}}\left(\frac{\hbar \bar{q}^{2}}{2 m^{*} \omega_{c}}\right) \frac{f\left(\varepsilon\left(n^{\prime}, k_{y}-q_{y}\right)\right)-f\left(\varepsilon\left(n, k_{y}\right)\right)}{\varepsilon\left(n^{\prime}, k_{y}-q_{y}\right)-\varepsilon\left(n, k_{y}\right)+\hbar \omega+i \hbar \eta},
$$

where

$$
C_{n n^{\prime}}(x)=\frac{n_{2} !}{n_{1} !} e^{-x} x^{n_{1}-n_{2}}\left[L_{n_{2}}^{n_{1}-n_{2}}(x)\right]^{2}
$$

with $n_{1}=\max \left(n, n^{\prime}\right), n_{2}=\min \left(n, n^{\prime}\right), f(\varepsilon)$ is the Fermi-Dirac distribution function, $\bar{q}$ is the $2 \mathrm{D}$ wave number, $A$ is the area of the system, $x=\frac{\hbar \bar{q}^{2}}{2 m^{*} \omega_{c}}$ and $L_{n}^{l}(x)$ are associated Laguerre polynomials. The density-density response function of the interacting system can be expressed as

$$
\Pi(\bar{q}, \omega)=\frac{\Pi_{0}(\bar{q}, \omega)}{1-v_{c}(\bar{q}) \Pi_{0}(\bar{q}, \omega)}
$$

with $v_{c}(\bar{q})=\frac{2 \pi e^{2}}{k \bar{q}}$ is the 2-D Coulomb potential, $k$ is the background dielectric constant. Using the transformation $k_{y} \rightarrow-k_{y}$, realizing that $\varepsilon\left(n, k_{y}\right)$ is an even function of $k_{y}$, interchanging $n \leftrightarrow n^{\prime}$ we can write the non-interacting density-density response function given 
by equation (5) as

$$
\begin{aligned}
\Pi_{0}(\bar{q}, \omega) & =\frac{m^{*} \omega_{c}}{\pi \hbar a} \sum_{n, n^{\prime}} C_{n n^{\prime}}\left(\frac{\hbar \bar{q}^{2}}{2 m^{*} \omega_{c}}\right) \int_{0}^{a} d x_{0}\left[f\left(\varepsilon\left(n, x_{0}+x_{0}^{\prime}\right)-f\left(\varepsilon\left(n^{\prime}, x_{0}\right)\right)\right]\right. \\
& \times\left[\varepsilon\left(n, x_{0}+x_{0}^{\prime}\right)-\varepsilon\left(n^{\prime}, x_{0}\right)+\hbar \omega+i \hbar \eta\right]^{-1} .
\end{aligned}
$$

In writing the above equation we converted the $k_{y}$-sum into an integral over $x_{0}$ and $x_{0}^{\prime}=$ $-\frac{\hbar q_{y}}{m^{*} \omega_{c}}$.

\section{MAGNETOPLASMONS MODES OF MM2DEG AND COMPARISON WITH EM2DEG AT ZERO TEMPERATURE}

The plasma modes are obtained from the roots of the longitudinal dispersion relation from Eqs. $(6,7)$

$$
1-v_{c}(\bar{q}) \operatorname{Re} \Pi_{0}(\bar{q}, \omega)=0
$$

along with the condition $\operatorname{Im} \Pi_{0}(\bar{q}, \omega)=0$ to ensure long-lived excitations. The roots of Eq. (8) give the plasma modes of a MM2DEG as

$$
1=\frac{2 \pi e^{2}}{\kappa \bar{q}} \frac{2}{\pi l^{2}} \sum_{n, n^{\prime}} C_{n n^{\prime}}(x)\left\{I_{1}\left(n, n^{\prime}, x_{0}^{\prime} ; \omega\right)+I_{1}\left(n, n^{\prime}, x_{0}^{\prime} ;-\omega\right)\right\},
$$

with

$$
I_{1}\left(n, n^{\prime}, x_{0}^{\prime} ; \omega\right)=P \int_{0}^{a} d x_{0} \frac{f\left(\varepsilon\left(n, x_{0}\right)\right)}{\varepsilon\left(n^{\prime}, x_{0}+x_{0}^{\prime}\right)-\varepsilon\left(n, x_{0}+x_{0}^{\prime}\right)+\hbar \omega} .
$$

and $\mathrm{P}$ is the principal value. From here on we will only show the dependence of $I_{1}$ on $\omega$ and suppress the rest such that $I_{1}\left(n, n^{\prime}, x_{0}^{\prime} ; \omega\right) \rightarrow I_{1}(\omega)$.

$\mathrm{SdH}$ and Weiss oscillations are found to occur in the magnetoconductivity of both electric and magnetically modulated 2DEG. These transport measurements can be explained without taking into account electron-electron interactions. In order to investigate collective excitations of the system such as magnetoplasmons it is essential to consider electron-electron interactions. Magnetoplasmons arise due to the coherent motion of electrons as a result of electron-electron interactions. Two types of magnetoplasmons can be identified: Those arising from electronic transitions involving different Landau bands (inter Landau band plasmons) and those within a single Landau band (intra Landau band plasmons). Inter-Landau band plasmons involve the local 1D magnetoplasma mode and the Bernstein-like plasma 
resonances [35, 36], all of which involve excitation energies greater than the Landau-band separation $\left(\sim \hbar \omega_{c}\right)$. On the other hand, intra-Landau band magnetoplasmons resonate at energies comparable to the bandwidths, and the existence of this new class of modes is due to finite width of the Landau levels. In a MM2DEG considered here, the Landau bandwidth $\left(\sim\left|G_{n}\right|\right)$ oscillates as a function of the band index $n$, since $L_{n}(u), L_{n}^{l}(u)$ are oscillatory functions of the index $n$. Such oscillating bandwidths affect the plasmon spectrum of the intra-Landau band type, when Landau-band separation is larger than the bandwidth as is the case considered here, resulting in magnetic Weiss oscillations similar to the electric Weiss oscillations found in the electrically modulated system. These oscillations are accompanied by SdH type of oscillatory behavior [29, 37]. Both these oscillations are periodic as a function of inverse magnetic field $(1 / B)$ but occur with different periods and amplitudes. As we show below, Weiss oscillations in the magnetoplasmon spectrum of a MM2DEG differ in phase and amplitude with those of an EM2DEG[29].

We now examine the inter-Landau-band transitions. These transitions occur between different Landau bands so we consider $n \neq n^{\prime}$ in Eq.(10) which yields

$$
I_{1}(\omega)=\frac{f(\varepsilon(n))}{(\hbar \omega-\Delta)}
$$

where $\Delta=\left(\varepsilon(n)-\varepsilon\left(n^{\prime}\right)\right)$ with $\varepsilon(n)=\left(n+\frac{1}{2}\right) \hbar \omega_{c}$, which permits us to write the following term in $\mathrm{Eq}(9)$ as

$$
\left(I_{1}(\omega)+I_{1}(-\omega)\right)=2 \frac{\Delta f(\varepsilon(n))}{(\hbar \omega)^{2}-(\Delta)^{2}} .
$$

Next, we consider the coefficient $C_{n n^{\prime}}(x)$ in Eq.(9) and expand it to lowest order in its argument (low wave-number expansion). In this case, we are only considering the $n^{\prime}=n \pm 1$ terms. The inter-Landau band plasmon modes under consideration arise from neighboring Landau bands. Hence for $n^{\prime}=n+1$ and $x \ll 1$, using the following associated Laguerre polynomial expansion [38] $L_{n}^{l}(x)=\sum_{m=0}^{n}(-1)^{m} \frac{(n+l) !}{(l+m) !(n-m) !} \frac{x^{m}}{m !}$ for $l>0$ and retaining the first term in the expansion for $x \ll 1, C_{n n^{\prime}}(x)$ reduces to

$$
C_{n, n+1}(x) \rightarrow(n+1) x,
$$

and for $n^{\prime}=n-1$ and $x \ll 1$, it reduces to

$$
C_{n, n-1}(x) \rightarrow n x
$$


Substitution of equations $(11)$ and $(12,13)$ into equation $(9)$ and replacing $x=\frac{\hbar \bar{q}^{2}}{2 m^{*} \omega_{c}}$ yields

$$
1=\frac{2 \pi e^{2}}{k m^{*}} \bar{q} \frac{1}{\omega^{2}-\omega_{c}^{2}}\left(\frac{m \omega_{c}}{\pi \hbar} \sum_{n} f(\varepsilon(n)) .\right.
$$

The term in parenthesis is easily recognized as the unmodulated particle density $n_{D}=$ $\frac{m \omega_{c}}{\pi \hbar} \sum_{n} f(\varepsilon(n))$, where the summation is over all occupied Landau bands. Defining the plasma frequency through $\omega_{p, 2 D}^{2}=\frac{2 \pi n_{D} e^{2}}{k m} \bar{q}$, we obtain the inter-Landau-band plasmon dispersion relation $1=\frac{\omega_{p, 2 D}^{2}}{\omega^{2}-\omega_{c}^{2}}$ or

$$
\omega^{2}=\omega_{c}^{2}+\omega_{p, 2 D}^{2}
$$

here $\omega$ is the well-known local 2D principal plasma frequency. Since we are interested in the modulation induced effects on the magnetoplasmons in this system, our focus will be on the intra-Landau band magnetoplasmons as the dispersion relation for inter-Landau band magnetoplasmons have been discussed and results displayed in [12, 29].

For the intra-Landau-band excitations spectrum, we need to consider transitions within a Landau miniband, i.e. $n=n^{\prime}, \varepsilon\left(n^{\prime}\right)-\varepsilon(n)=0$ and $C_{n n^{\prime}}(x) \rightarrow 1$. An analytical expression of the intra-Landau band plasmon energy $\hbar \tilde{\omega}$ can be obtained

$$
\hbar^{2} \sim^{2}=\frac{16 e^{2}}{k \bar{q} \pi} \frac{m^{*} \omega_{c}}{\hbar a} \sin ^{2}\left(\frac{\pi}{a}\left(x_{0}^{\prime}\right)\right) \times A_{n},
$$

where

$$
A_{n}=\sum_{n} G_{n} \times \int_{0}^{a / 2} d x_{0} f\left(\varepsilon\left(n, x_{0}\right)\right) \cos \left(K x_{0}\right) .
$$

At zero temperature $(T=0)$,

$$
\hbar^{2} \sim^{2}=\frac{8 e^{2}}{k \bar{q}} \frac{m^{*} \omega_{c}}{\pi \hbar} \sin ^{2}\left(\frac{\pi}{a}\left(x_{0}^{\prime}\right)\right) \times \sum_{n}\left|G_{n}\right| \sqrt{1-\Delta_{n}^{2}} \theta\left(1-\Delta_{n}\right),
$$

with $\Delta_{n}=\left|\frac{\varepsilon_{F}-\varepsilon(n)}{G_{n}}\right|, \theta(x)$ the Heaviside unit step function. If we replace the magnetic modulation term $\left(G_{n}\right)$ by the electric one, Eq. (16) has the same structure as Eq. (8) of [29] that pertains to electric modulation. The above expression for $\hbar \tilde{\omega}$ has been obtained under the condition $\hbar \omega>>\left|\varepsilon\left(n, x_{0}+x_{0}^{\prime}\right)-\varepsilon\left(n, x_{0}\right)\right|$ as $x_{0}^{\prime} \rightarrow 0$ which leads to a relation between the energy and the Landau level broadening $\hbar \omega>>\left|2 G_{n} \sin \left(\frac{\pi}{a} x_{0}^{\prime}\right) \sin \left[\left(\frac{2 \pi}{a}\right)\left(x_{0}+\frac{x_{0}^{\prime}}{2}\right)\right]\right|$. This ensures that $\operatorname{Im} \Pi_{0}(\bar{q}, \omega)=0$ and the intra-Landau-band magnetoplasmons are undamped. For a given $G_{n}$, this can be achieved with a small but nonzero $q_{y}$ (recall that $x_{0}^{\prime}=-\frac{\hbar q_{y}}{m^{*} \omega_{c}}$ ). 
In general, the inter- and intra-Landau-band modes are coupled for arbitrary magnetic field strengths. The general dispersion relation is :

$$
1=\frac{\omega_{p, 2 D}^{2}}{\omega^{2}-\omega_{c}^{2}}+\frac{\tilde{\omega}^{2}}{\omega^{2}}
$$

This equation yields two modes which are given by

$$
\begin{aligned}
\omega_{ \pm}^{2} & =\frac{1}{2}\left(\omega_{c}^{2}+\omega_{p, 2 D}^{2}+\tilde{\omega}^{2}\right) \pm \frac{1}{2}\left\{\left(\omega_{c}^{2}+\omega_{p, 2 D}^{2}+\tilde{\omega}^{2}+2 \omega_{c} \tilde{\omega}\right)\right. \\
& \left.\times\left(\omega_{c}^{2}+\omega_{p, 2 D}^{2}+\tilde{\omega}^{2}-2 \omega_{c} \tilde{\omega}\right)\right\}^{1 / 2}
\end{aligned}
$$

which reduces to

$$
\omega_{+}^{2}=\omega_{c}^{2}+\omega_{p, 2 D}^{2}
$$

and

$$
\omega_{-}^{2}=\tilde{\omega}^{2}
$$

with corrections of order $\tilde{\omega}^{2} / \omega_{c}^{2}$ and $\tilde{\omega}^{2} / \omega_{p, 2 D}^{2}$. So long as $\left|G_{n}\right|<\hbar \omega_{c}$, mixing of the interand intra-band modes is small. Only the intra-Landau-band mode $(\hbar \tilde{\omega})$ will be excited in the frequency regime $\hbar \omega_{c}>\hbar \omega \sim\left|G_{n}\right|$.

The intra-Landau-band plasma energy given by equation (11) is shown graphically in Fig.(1) as a function of $1 / B$. The parameters used are [5, 7, 8, 26, 29, 30, 31]: $m^{*}=0.07 m_{e}$, $k=12, n_{D}=3.16 \times 10^{15} \mathrm{~m}^{-2}, a=380 \mathrm{~nm}$. We also take $q_{x}=0$ and $q_{y}=0.01 k_{F}$, with $k_{F}=\left(2 \pi n_{D}\right)^{1 / 2}$ being the Fermi wave number of the unmodulated 2DEG in the absence of magnetic field. In numerical evaluation we have taken the sum over thirty Landau levels which ensures convergence of numerical results. Numerical evaluation of the dispersion relation was performed in Mathematica. In Fig(1), modulation induced oscillations in the intra-Landau band mode are apparent, superimposed on SdH-type oscillations. These oscillations are periodic as a function of inverse magnetic field $(1 / B)$. To gain further insight into the results presented in Fig.(1), we consider equation (16). In the regime $\hbar \omega_{c}>\left|G_{n}\right|$, the unit step function vanishes for all but the highest occupied Landau band, corresponding , say, to the band index $N$. The sum over $n$ is trivial and plasma energy is given as $\hbar \tilde{\omega}=\left|G_{N}\right|^{1 / 2}\left(1-\Delta_{N}^{2}\right)^{1 / 4} \theta\left(1-\Delta_{N}\right)$. The analytic structure primarily responsible for the $\mathrm{SdH}$ type of oscillations is the function $\theta\left(1-\Delta_{N}\right)$, which jumps periodically from zero (when the Fermi level is above the highest occupied Landau band) to unity (when the Fermi level is contained with in the highest occupied Landau band). On the other hand, the 
periodic modulation of the amplitude of the $\mathrm{SdH}$ type oscillations is due to the oscillatory nature of the factor $\left|G_{N}\right|^{1 / 2}$, which has been shown to exhibit commensurability oscillations [6, 19, 21, 31]. In the same figure, we also show the intra-Landau band plasma energy for the electrically modulated 2DEG[29]. The magnetoplasmons spectrum of the MM2DEG at a specific Landau level is minimum when the corresponding spectrum for the EM2DEG is maximum, this confirms that magnetic oscillations in the two system are out of phase. To have comparable results for the two systems, at zero temperature, the strength of magnetic modulation potential, $\hbar \omega_{0}$, has to be $\sim 8.5$ times smaller than that of the electric modulation potential, $V_{0}$. This can be understood if we realize that this constraint arises due to the step function $\theta\left(1-\Delta_{N}\right)$ appearing in the dispersion relation at zero temperature. Therefore, comparable results for the two systems when they are subjected to modulation of equal strength requires that we carry out a finite temperature calculation to avoid the constraint imposed by the step function at zero temperature.

\section{TEMPERATURE DEPENDENT MAGNETOPLASMONS MODE OF MM2DEG AND COMPARISON WITH EM2DEG}

For the finite temperature calculation of the intra-landau band plasma energy, we invoke the condition of weak modulation and perform the following expansion in equation (15)

$$
f\left(\varepsilon\left(n, x_{0}\right)\right) \simeq f(\varepsilon(n))+G_{n} f^{\prime}(\varepsilon(n)) \cos \left(K x_{0}\right),
$$

where $f^{\prime}(x)=\frac{d}{d x} f(x)$ is the derivative of the Fermi-Dirac distribution function. With the substitution of this expansion in equation (15), the integral over $x_{0}$ contains two terms. The integral over $x_{0}$ of the first term vanishes and the integral of the second term yields intra-Landau band dispersion relation

$$
\hbar^{2} \tilde{\omega}^{2}=\frac{4 e^{2} m^{*} \omega_{c}}{k \bar{q} \pi \hbar} \sin ^{2}\left[\frac{\pi}{a}\left(x_{0}^{\prime}\right)\right] \times B_{n},
$$

where

$$
B_{n}=\sum_{n} G_{n}^{2} \times\left[-f^{\prime}(\varepsilon(n))\right] .
$$

To facilitate comparison of the above dispersion relation with the results obtained for an electrically modulated 2DEG we will determine the asymptotic expressions of intra-Landau 
band magnetoplasmon spectrum, where analytic results in terms of elementary functions can be obtained. Moreover, these asymptotic expressions will allow us to identify terms responsible for $\mathrm{SdH}$ and Weiss oscillations and how they are affected by temperature.

The asymptotic expression can be obtained by using the following asymptotic expression for the Laguerre polynomials [5, 7, 8, 26]

$$
\exp ^{-u / 2} L_{n}(u) \rightarrow \frac{1}{\sqrt{\pi \sqrt{n u}}} \cos \left(2 \sqrt{n u}-\frac{\pi}{4}\right) .
$$

Note that the above asymptotic expression for $L_{n}(u)$ is valid for $n \gg 1$, at low magnetic fields when many Landau Levels are filled. We now take the continuum limit:

$$
n-->\frac{\varepsilon(n)}{\hbar \omega_{c}}, \sum_{n=0}^{\infty}-->\int_{0}^{\infty} \frac{d \varepsilon}{\hbar \omega_{c}} .
$$

In the asymptotic limit, $B_{n}$ that appears in equation (18) can be written as

$$
B_{n}=\frac{\hbar^{2} \omega_{0}^{2}}{\pi \hbar \omega_{c}} \sqrt{\frac{\hbar \omega_{c}}{u}}\left(\frac{a K_{F}}{2 \pi}\right)^{2} \int_{0}^{\infty} \frac{d \varepsilon}{\sqrt{\varepsilon}} \frac{\beta g(\varepsilon)}{[g(\varepsilon)+1)]^{2}} \sin ^{2}\left(2 \sqrt{n u}-\frac{\pi}{4}\right)
$$

where $g(\varepsilon)=\exp \left[\beta\left(\varepsilon-\varepsilon_{F}\right)\right], \beta=\frac{1}{K_{B} T}$.

Now assuming that temperature is low such that $\beta^{-1} \ll \varepsilon_{F}$ and replacing $\varepsilon=\varepsilon_{F}+s \beta^{-1}$, we can express the above integral as

$$
B_{n}=\frac{\hbar^{2} \omega_{0}^{2}}{\pi \sqrt{u \hbar \omega_{c} \varepsilon_{F}}}\left(\frac{a K_{F}}{2 \pi}\right)^{2} \int_{0}^{\infty} d s \frac{e^{s}}{\left.\left[e^{s}+1\right)\right]^{2}} \sin ^{2}\left(2 \sqrt{\frac{u \varepsilon_{F}}{\hbar \omega_{c}}}-\frac{\pi}{4}+\sqrt{\frac{u}{\hbar \omega_{c} \varepsilon_{F}}} s \beta^{-1}\right)
$$

with the result

$$
B_{n}=\frac{\hbar^{2} \omega_{0}^{2}}{2 \pi \sqrt{u \hbar \omega_{c} \varepsilon_{F}}}\left(\frac{a K_{F}}{2 \pi}\right)^{2}\left[1-A\left(\frac{T}{T_{a}}\right)+2 A\left(\frac{T}{T_{a}}\right) \sin ^{2}\left[2 \sqrt{\frac{u \varepsilon_{F}}{\hbar \omega_{c}}}-\frac{\pi}{4}\right]\right]
$$

where $T_{a}$ is the characteristic damping temperature of Weiss oscillationgiven by $k_{B} T_{a}=$ $\frac{\hbar \omega_{c} a K_{F}}{4 \pi^{2}}, \frac{T}{T_{a}}=\frac{4 \pi^{2} k_{B} T}{\hbar \omega_{c} a K_{F}}$ and $A(x)=\frac{x}{\sinh (x)}-{ }^{(x-->\infty)}->=2 x e^{-x}$.

From equation(18), the asymptotic expression for intra-Landau band plasmon spectrum is obtained

$$
\begin{aligned}
\hbar^{2} \widetilde{\omega}^{2} & =\frac{4 \hbar^{2} \omega_{0}^{2} e^{2} m^{*} \omega_{c}}{k \bar{q} \hbar 2 \pi^{2} \sqrt{u \hbar \omega_{c} \varepsilon_{F}}}\left(\frac{a K_{F}}{2 \pi}\right)^{2} \sin ^{2}\left[\frac{\pi}{a}\left(x_{0}^{\prime}\right)\right] \\
& \times\left[1-A\left(\frac{T}{T_{a}}\right)+2 A\left(\frac{T}{T_{a}}\right) \sin ^{2}\left(2 \sqrt{\frac{u \varepsilon_{F}}{\hbar \omega_{c}}}-\frac{\pi}{4}\right)\right]
\end{aligned}
$$


The above expression is not able to account for the SdH type of oscillations in the magnetoplasmon spectrum. These oscillations can be accounted for by expressing the density of states (in the absence of disorder) [5] as

$$
D(\varepsilon)=\frac{m^{*}}{\pi \hbar}\left(1-2 \cos \left[\frac{2 \pi \varepsilon}{\hbar \omega_{c}}\right]\right)
$$

and inserting the continuum approximation as $\sum_{n=0}^{\infty}-->2 \pi l^{2} \int_{0}^{\infty} D(\varepsilon) d \varepsilon$, this yields the asymptotic expression for the intra-Landau band magnetoplasmon dispersion relation for MM2DEG

$$
\begin{aligned}
\hbar^{2} \sim^{2} & =\frac{4 \hbar^{2} \omega_{0}^{2} e^{2} m^{*} \omega_{c}}{k \bar{q} \hbar 2 \pi^{2} \sqrt{u \hbar \omega_{c} \varepsilon_{F}}}\left(\frac{a K_{F}}{2 \pi}\right)^{2} \sin ^{2}\left[\frac{\pi}{a}\left(x_{0}^{\prime}\right)\right] \times\left\{\left[1-A\left(\frac{T}{T_{a}}\right)\right.\right. \\
& \left.\left.+2 A\left(\frac{T}{T_{a}}\right) \sin ^{2}\left(2 \sqrt{\frac{u \varepsilon_{F}}{\hbar \omega_{c}}}-\frac{\pi}{4}\right)\right]-4 A\left(\frac{T}{T_{s}}\right) \cos \left[\frac{2 \pi \varepsilon_{F}}{\hbar \omega_{c}}\right] \sin ^{2}\left[2 \sqrt{\frac{u \varepsilon_{F}}{\hbar \omega_{c}}}-\frac{\pi}{4}\right]\right\}
\end{aligned}
$$

where $\frac{T}{T_{s}}=\frac{2 \pi^{2} k_{B} T}{\hbar \omega_{c}}, T_{s}$ defines the characteristic damping temperature of the SdH oscillations in the magnetoplasmon spectrum of MM2DEG.

Following the same approach as discussed above for MM2DEG, we can obtain the intraLandau band magnetoplasmon spectrum for EM2DEG

$$
\hbar^{2} \tilde{\omega}^{2}=\frac{4 e^{2} m^{*} \omega_{c}}{\hbar k \bar{q} \pi} \sin ^{2}\left[\frac{\pi}{a}\left(x_{0}^{\prime}\right)\right] \times B_{n},
$$

where $B_{n}=\sum F_{n}^{2} \times\left[-f^{\prime}(\varepsilon(n))\right]$, and $F_{n}=V_{0} e^{-u / 2} L_{n}(u)$ is the modulation width of the EM2DEG with $V_{0}$ the amplitude of electric modulation. The corresponding asymptotic result for EM2DEG is

$$
\begin{aligned}
\hbar^{2} \tilde{\omega}^{2} & =\frac{4 V_{0}^{2} e^{2} m^{*} \omega_{c}}{k \bar{q} 2 \pi^{2} \hbar \sqrt{u \hbar \omega_{c} \varepsilon_{F}}} \sin ^{2}\left(\frac{\pi}{a}\left(x_{0}^{\prime}\right)\right) \times\left\{\left[1-A\left(\frac{T}{T_{a}}\right)\right.\right. \\
& \left.\left.+2 A\left(\frac{T}{T_{a}}\right) \cos ^{2}\left(2 \sqrt{\frac{u \varepsilon_{F}}{\hbar \omega_{c}}}-\frac{\pi}{4}\right)\right]-4 A\left(\frac{T}{T_{s}}\right) \cos \left[\frac{2 \pi \varepsilon_{F}}{\hbar \omega_{c}}\right] \cos ^{2}\left[2 \sqrt{\frac{u \varepsilon_{F}}{\hbar \omega_{c}}}-\frac{\pi}{4}\right]\right\}
\end{aligned}
$$

The intra-landau band plasmon dispersion relations obtained for the MM2DEG and the EM2DEG systems given by equations $(25,27)$ allow us to identify the terms responsible for Weiss and $\mathrm{SdH}$ oscillations. Moreover, the characteristic damping temperatures appearing in these expressions carry the effects of temperature on these oscillations. Comparing equations $(25,27)$, the following differences can be highlighted:

1) Amplitude of the oscillations (Weiss and $\mathrm{SdH}$ ) are larger by the factor $\frac{a K_{F}}{2 \pi}$ in MM2DEG compared to those of EM2DEG. 
2) The factor $\sin ^{2}\left[2 \sqrt{\frac{u \varepsilon_{F}}{\hbar \omega_{c}}}-\frac{\pi}{4}\right]$ that appears in equation (25) for MM2DEG and the corresponding factor $\cos ^{2}\left[2 \sqrt{\frac{u \varepsilon_{F}}{\hbar \omega_{c}}}-\frac{\pi}{4}\right]$ in equation (27) for EM2DEG results in a $\pi / 2$ phase difference in the oscillations in the magnetoplasmon spectrum of the two systems

Since equations $(25,27)$ are the key results of this work, we show the intra-Landau band magnetoplasmon energy for both the magnetically and electrically modulated systems as a function of inverse magnetic field in Fig.(2). The results presented are for equal strength of the two modulations which is taken to be $V_{0}=\hbar \omega_{0}=1 \mathrm{meV}$. The temperature is $0.4 \mathrm{~K}$. Rest of the parameters are the same as the case for the zero temperature results presented in Fig.(1). We observe the modulation induced effects in the intra-Landau band mode, Weiss oscillations modulating the $\mathrm{SdH}$ oscillations in the magnetoplasmon spectrum. From the figure, we see that the amplitude of Weiss oscillations in the magnetically modulated system is greater by a factor of $\sim 8.5$ compared to the electrically modulated system. This can be seen from equations $(25,27)$ where the difference in the amplitudes is the factor $\frac{a K_{F}}{2 \pi}$ and it is $\sim 8.5$ for the parameters that we have used. Therefore, in the magnetically modulated system the amplitude is larger by this factor compared to the electrically modulated system. We also observe that Weiss oscillations in the MM2DEG are out of phase by $\pi / 2$ compared to those in EM2DEG. To see the effects of temperature on Weiss and SdH oscillations in a MM2DEG, we plot the intra-Landau band plasmon energy for a MM2DEG as a function of inverse magnetic field at two different temperatures in Fig.(3). The modulation strength is $1 \mathrm{meV}$. The results are shown at the following two temperatures: $0.3 \mathrm{~K}$ and $3 \mathrm{~K}$. The $\mathrm{SdH}$ oscillations are completely damped at $3 \mathrm{~K}$ whereas Weiss oscillations persist at this temperature. Eqs. $(25,27)$ also allow us to determine the temperature scales for damping of Weiss and SdH oscillations in the magnetoplasmon spectrum. For a MM2DEG, from Eq.(25), $\frac{T_{a}}{T_{s}}=\frac{a K_{F}}{2} \gg 1$; e.g., $n_{D}=3.16 \times 10^{15} \mathrm{~m}^{-2}$ and $a=380 \mathrm{~nm}$, we have $\frac{T_{a}}{T_{s}}=27$ for the experimentally relevant parameters considered here. Hence, SdH oscillations are completely damped at a much lower temperature compared to Weiss oscillations. These results are consistent with and complement those obtained from electron transport studies of a magnetically modulated 2DEG [5, 6, 17, 8, 19, 20, 21, 26, 27]. 


\section{MAGNETOPLASMON SPECTRUM WITH PERIODIC ELECTRIC AND MAGNETIC MODULATION: IN-PHASE}

In this section, we calculate the magnetoplasmon spectrum when electric and magnetic modulations are in-phase. We take the magnetic modulation to have the same phase as given in the previous section with the in-phase electric modulation. The energy eigenvalues are [5, 26]

$$
\varepsilon\left(n, x_{0}\right)=(n+1 / 2) \hbar \omega_{c}+\left(G_{n}+F_{n}\right) \cos \left(K x_{o}\right)
$$

and the bandwidth can be written as

$$
\Delta(\text { in }- \text { phase })=\frac{2 \hbar \omega_{0} a k_{F} \times \sqrt{1+\delta^{2}}}{2 \pi \sqrt{\pi \sqrt{n u}}} \times \sin \left(2 \sqrt{n u}-\frac{\pi}{4}+\Phi\right)
$$

where the ratio between the two modulation strengths $\delta=\frac{2 \pi V_{0}}{\hbar \omega_{0} a k_{F}}=\tan (\Phi)$. The flat band condition from the above equation is $2 \sqrt{n u}-\frac{\pi}{4}+\Phi=i \pi$ where $i$ is an integer. This condition

can also be expressed as $\frac{\sqrt{2 n}}{a} l=i+\frac{1}{4}-\frac{\Phi}{\pi}$, where $n=n_{F}=\frac{\varepsilon_{F}}{\hbar \omega_{c}}-\frac{1}{2}$ is the highest Fermi integer. We see that the flat band condition in this case depends on the relative strength of the two modulations.

Following the same approach as discussed in the previous section for the MM2DEG, we can obtain the intra-Landau band magnetoplasmon spectrum in the presence of in phase modulations as

$$
\hbar^{2} \tilde{\omega}^{2}=\frac{4 e^{2} m^{*} \omega_{c}}{k \bar{q} \pi \hbar} \sin ^{2}\left[\frac{\pi}{a}\left(x_{0}^{\prime}\right)\right] \times I_{n}
$$

where

$$
I_{n}=\sum_{n}\left(G_{n}+F_{n}\right)^{2} \times\left[-f^{\prime}(\varepsilon(n))\right]
$$

In Fig.(4) we show the in-phase magnetoplasmon spectrum (the magnetic and the electric modulations are in-phase) $\hbar \tilde{\omega}$ given by Eq.(30) as a function of the inverse magnetic field for temperature $T=0.3 \mathrm{~K}$, electron density $n_{e}=3 \times 10^{11} \mathrm{~cm}^{-2}$, the period of modulation $a=380 \mathrm{~nm}$. The strength of the electric modulation $V_{0}=0.2 \mathrm{meV}$ whereas $B_{0}=0.02 \mathrm{~T}$ which corresponds to $\hbar \omega_{0}=0.03 \mathrm{meV}$. In the same figure we have also shown the magnetoplasmon spectrum when either the magnetic or electric modulation alone is present. The $\frac{\pi}{2}$ phase difference in the bandwidths results in the same phase difference appearing in the magnetoplason spectrum for electric and magnetic modulations as can be seen in the figure. To better understand the effects of in-phase modulations on the magnetoplasmon spectrum 
we consider the asymptotic expression of the magnetoplasmon spectrum given by Eq.(30). The asymptotic expression is given by

$$
\begin{aligned}
\hbar^{2} \tilde{\omega}^{2} & =\frac{2 V_{0}^{2} e^{2} m^{*} \omega_{c} \delta^{-2}}{k \bar{q} 2 \pi^{2} \hbar \sqrt{u \hbar \omega_{c} \varepsilon_{F}}} \sin ^{2}\left(\frac{\pi}{a}\left(x_{0}^{\prime}\right)\right) \times\left(1+\delta^{2}\right)\left\{\left[1-A\left(\frac{T}{T_{a}}\right)\right.\right. \\
& \left.+\left(2 A\left(\frac{T}{T_{a}}\right)-4 A\left(\frac{T}{T_{s}}\right) \cos \left[\frac{2 \pi \varepsilon_{F}}{\hbar \omega_{c}}\right]\right) \sin ^{2}\left[2 \sqrt{\frac{u \varepsilon_{F}}{\hbar \omega_{c}}}-\frac{\pi}{4}+\Phi\right]\right\}
\end{aligned}
$$

From the asymptotic expression given by Eq.(31), we observe that in the presence of inphase electric and magnetic modulatio the magnetoplasmon energy acquires a dependence on the phase factor $\Phi$ and $\delta$ which depend on the relative modulation strengths. The shift in the Weiss oscillations when in-phase electric and magnetic modulations are present can be seen in Fig.(5). How the Weiss oscillations are affected as $\Phi$ as well as the magnetic field is varied can be seen in Fig.(5). The results shown are for a fixed magnetic modulation of strength $\hbar \omega_{0}=0.03 \mathrm{meV}$ and the electric modulation is varied. The change in $V_{0}$ results in a corresponding change in both $\delta$ and $\Phi$. From Fig.(5), we observe that the position of the extrema in the magnetoplasmon spectrum as a function of the inverse magnetic field depend on the relative strength of the modulations.

The effects of electric and magnetic modulations that are out-of-phase on the magnetoplasmon spectrum can be better appreciated if we consider the asymptotic expression in this case. This is taken up in the next section.

\section{MAGNETOPLASMONS WITH PERIODIC ELECTRIC AND MAGNETIC MODULATION: OUT-OF-PHASE}

In this section, we calculate the magnetoplasmon spectrum when electric and magnetic modulations are out of phase by $\pi / 2$. We consider magnetic modulation out of phase with the electric one: We take the electric modulation to have the same phase as given in the previous section with the $\pi / 2$ phase difference incorporated in the magnetic field. The energy eigenvalues are[5, 26]

$$
\varepsilon\left(n, x_{0}\right)=(n+1 / 2) \hbar \omega_{c}+\sin \left(K x_{0}\right) G_{n}+F_{n} \cos \left(K x_{0}\right),
$$

and the bandwidth is

$$
\Delta(\text { out of phase })=\frac{2 \hbar \omega_{0} a k_{F}}{2 \pi \sqrt{\pi \sqrt{n u}}} \times \sqrt{\delta^{2}+\left(1-\delta^{2}\right) \sin \left(2 \sqrt{n u}-\frac{\pi}{4}\right)} .
$$


The term responsible for Weiss oscillations is the $\sin \left(2 \sqrt{n u}-\frac{\pi}{4}\right)$ term under the square root which can be readily seen by considering the large $n$ limit of the bandwidth. Therefore for $\delta= \pm 1$ Weiss oscillations are no longer present in the bandwidth.

Following the same approach discussed in the previous section for MM2DEG, we can obtain the intra-Landau band magnetoplasmon spectrum in the presence of out of phase modulations as

$$
\hbar^{2} \tilde{\omega}^{2}=\frac{4 e^{2} m^{*} \omega_{c}}{k \bar{q} \pi \hbar} \sin ^{2}\left[\frac{\pi}{a}\left(x_{0}^{\prime}\right)\right] \times O_{n}
$$

where

$$
O_{n}=\sum_{n}\left(G_{n}^{2}+F_{n}^{2}\right) \times\left[-f^{\prime}(\varepsilon(n))\right]
$$

The asymptotic expression in the presence of both electric and magnetic modulations that are out of phase is obtained by substituting the asymptotic expressions for the Laguerre polynomials and converting the sum into integration with the result

$$
\begin{aligned}
\hbar^{2} \sim^{2} & =\frac{2 V_{0}^{2} e^{2} m^{*} \omega_{c} \delta^{-2}}{k \bar{q} 2 \pi^{2} \hbar \sqrt{u \hbar \omega_{c} \varepsilon_{F}}} \sin ^{2}\left(\frac{\pi}{a}\left(x_{0}^{\prime}\right)\right) \times\left\{2 \delta^{2}+\left(1-\delta^{2}\right)\left[1-A\left(\frac{T}{T_{a}}\right)\right.\right. \\
& \left.\left.\left.+2 A\left(\frac{T}{T_{a}}\right) \sin ^{2}\left[2 \sqrt{\frac{u \varepsilon_{F}}{\hbar \omega_{c}}}-\frac{\pi}{4}\right]\right]-4 A\left(\frac{T}{T_{s}}\right) \cos \left[\frac{2 \pi \varepsilon_{F}}{\hbar \omega_{c}}\right]\right)\left[\delta^{2}-\left(\delta^{2}-1\right) \sin ^{2}\left[2 \sqrt{\frac{u \varepsilon_{F}}{\hbar \omega_{c}}}-\frac{\pi}{4}\right]\right]\right\}
\end{aligned}
$$

From the expression of the out-of-phase bandwidth given by Eq.(33) we find that Weiss oscillations in the bandwidth are absent for relative modulation strength $\delta= \pm 1$, the same is reflected in the magnetoplasmon spectrum as the term responsible for Weiss oscillations $\left(\sin ^{2}\left[2 \sqrt{\frac{u \varepsilon_{F}}{\hbar \omega_{c}}}-\frac{\pi}{4}\right]\right)$ vanishes for $\delta= \pm 1$ as can be seen from the above equation. Therefore the magnetoplasmon spectrum does not exhibit Weiss oscillations when the relative modulation strength $\delta= \pm 1$. The spectrum as a function of magnetic field when the electric and magnetic modulations are out-of-phase is shown in Fig.(6). The results shown are for a fixed magnetic modulation of strength $\hbar \omega_{0}=0.03 \mathrm{meV}$ and the electric modulation $V_{0}$ is allowed to vary between positive and negative values. The other parameters are the same as in Figs. $(4,5)$. As $V_{0}$ is varied there is a corresponding change in $\delta$. We find that the positions of the extrema of the spectrum as a function of the inverse magnetic field do not change as $\delta$ is varied since the phase factor $\Phi$ does not appear in the expression of magnetoplasmon spectrum when the two modulations are out of phase. It is also observed in Fig.(6) that there is a $\frac{\pi}{2}$ phase 
difference between the curves for $\delta \geq 1$ and $\delta<1$. The same behavior is observed in the bandwidth which is reflected in the magnetoplasmon spectrum.

\section{CONCLUSIONS}

In conclusion, we have determined the inter and intra-Landau band magnetoplasmon spectrum for a magnetically modulated two-dimensional electron gas in the presence of a perpendicular magnetic field. Our results show that magnetic Weiss oscillations occur in intra-Landau band magnetoplasmon spectrum. Their origin lies in the interplay of the two physical length scales of the system i.e. the modulation period, and cyclotron diameter at the Fermi level. When the strength of magnetic modulation potential is equal to the electric one, the magnetic Weiss oscillations in the magnetoplasmon spectrum are out of phase and occur with a larger amplitude compared to the electric Weiss oscillations in a 2DEG. These results also exhibit that the Weiss oscillations depend on the temperature much less than that of the SdH oscillations. We have determined the effects of both the electric and magnetic modulations on the magnetoplasmon spectrum of 2DEG. These oscillations are affected by the relative phase of the two modulations and positions of the extrema of the oscillations depend on the relative strength of the two modulations. We find complete suppression of Weiss oscillations for particular relative strength of the modulations when the modulations are out-of-phase.

K. Sabeeh would like to acknowledge the support of the Pakistan Science Foundation (PSF) through project No. C-QU/Phys (129). M. Tahir would like to acknowledge the support of the Pakistan Higher Education Commission (HEC).

*Electronic address: m.tahir06@imperial.ac.uk.

†Electronic address: ksabeeh@qau.edu.pk, kashifsabeeh@hotmail.com

[1] Izawa S, Katsamoto S, Endo A and Iye Y, J. Phys. Soc. Japan 64, 706-710 (1995).

[2] Ye P D, Weiss D, Gerhardts R R, Seeger M, von Klitzing K, Eberl K and Nickel H, Phys. Rev. Lett. 74, 3013-3016 (1995).

[3] Carmona H A, Geim A K, Nogaret A, Main P C, Foster T J, Henini M, Beaumont S P and Blamire M G, Phys. Rev. Lett. 74, 3009-3012 (1995). 
[4] Yagi R and Iye Y, J. Phys. Soc. Japan 62, 1279-1285 (1993).

[5] Peeters F M and Vasilopoulos P, Phys. Rev. B 47, 1466-1473 (1993).

[6] Wu Xiaoguang and Ulloa Sergio E, Solid State Commun. 82 945-949 (1992).

[7] Xue Deng Ping and Xiao Gang, Phys. Rev. B 45, 5986-5990 (1992).

[8] P. Vasilopoulos and F. M. Peeters, Superlattices Microstruct. 7, 393-395 (1990).; P. Vasilopoulos and F. M. Peeters, Physica Scripta. Vol. T39, 177-181 (1991).

[9] Yoshioka D and Iye Y, J. Phys. Soc. Japan 56, 448-451 (1987).

[10] J. M. Luttinger, Phys. Rev. 84, 814-817 (1951).

[11] Q. P. Li and S. Das Sarma, Phys. Rev. B 44, 6277-6238 (1991).

[12] S. M. Stewart and C. Zhang, J. Phys.: Condens. Matter 8, 6019-6033 (1996).

[13] P. Vasilopoulos, X. F. Wang and F. M. Peeters, Phys. Rev. B 70, 155312-1-8 (2004).; I. S. Ibrahim and F. M. Peeters, Phys. Rev. B 52, 17321-17334 (1995).

[14] Andrey Krakovsky, Phys. Rev. B 53, 8469-8472 (1996).; M. C. Chang and Q. Niu, Phys. Rev. B 52, 10843-10850 (1995).

[15] A. Y. Rom, Phys. Rev. B 55, 11025-11028 (1997).

[16] S. Cina, D. M. Whittaker, D. D. Arnone, T. Burke, H. P. Hughes, M. Leadbeater, M. Pepper, and D. A. Ritchie, Phys. Rev. Lett. 83, 4425-4428 (1999).

[17] V. Fessatidis, H. L. Cui, and P. Vasilopoulos, Superlattices and Microstruct. 23, 47-54 (1998).

[18] Mayumi Kato, Akira Endo, Makoto Sakairi, Shingo Katsumoto, and Yasuhiro Iye, J. Phys. Soc. Jpn. 68, 1492-1495 (1999).

[19] Tong-Zhong Li, Shi-Wei Gu, Xue-Hua Wang and Jian-Ping Peng, J. Phys.: Condens. Matter 8, 313-327 (1996).

[20] A. Manolescu and V. Gudmundsson, Superlattices Microstruct. 23, 1169-1180 (1998).

[21] X. Wu and S. E. Ulloa, Phys. Rev. B 47, 7182-7186 (1993).

[22] Iye Y, Endo A, Izawa S, Kato M and Katsumoto S, Physica B 227 122-126 (1996).

[23] J. E. Muller, Phys. Rev. Lett. 68, 385-388 (1992).

[24] X. G. Wu and S. E. Ulloa, Phys. Rev. B 47, 10 028-10031 (1993).

[25] Jirong Shi, F. M. Peeters, K. W. Edmonds, and B. L. Gallagher, Phys. Rev. B 66, 035328-1-9 (2002).

[26] F M Peeters, P Vasilopoulos, Jirong Shi, Journal of Physics Condensed Matter 14, 8803-8816 (2002). 
[27] K. W. Edmonds, B. L. Gallagher, P. C. Main, N. Overend, R. Wirtz, A. Nogaret, M. Henini, C. H. Marrows, B. J. Hickey, and S. Thoms, Phys. Rev. B 64, 041303-1-4 (2001).

[28] A. Matulis and F. M. Peeters, Phys. Rev. B 62, 91-94 (2000).

[29] H. L. Cui, V. Fessatidis, and N. J. M. Horing, Phys. Rev. Lett. 63, 2598-2601 (1989).

[30] W-M Que, G. Kirczenow, Phys. Rev. Lett. 62, 1687 (1989).

[31] Weiss D, Klitzing K V, Ploog K and Weiman G, Europhys. Lett. 8 179-184 (1989).; R. W. Winkler and J. P. Kotthaus, K. Ploog, Phys. Rev. Lett 62, 1177-1180 (1989).; Gerhardts R R, Weiss D and von Klitzing K, Phys. Rev. Lett. 62, 1173-1176 (1989).

[32] O. G. Balev, N. Studart, and P. Vasilopoulos, Phys. Rev. B 62, 15834 (2000),; M. S. Kushwaha, Surf. Sci. Rep. 41, 1-416 (2001), and references therein.

[33] Handbook of Mathematical Functions, edited by M. Abramowitz and I. A. Stegun (Dover, New York, 1972).

[34] H. Ehrenreich and M. H. Cohen, Phys. Rev. 115, 786-790 (1959).

[35] R. Krahne, M. Hochgräfe, Ch. Heyn, and D. Heitmann, Phys. Rev. B 61, R16319-R16322 (2000).

[36] Wei-ming Que and George Kirczenow, Phys. Rev. B 36, 6596-6601 (1987).

[37] T. Demel, D. Heitmann, P. Grambow, and K. Ploog, Phys. Rev. B 38, 12 732-12735 (1988).

[38] I. S. Gradshteyn and I. M. Ryzhik, Table of Integrals, Series and Products (Academic Press, New York, 1980). 